\title{
Related Risk Factors among Women Enrolled in a Community-Based Perinatal Care Program
}

\author{
Matt Hodges ${ }^{1}$, Qing Tang ${ }^{1,2}$, Debra Litzelman ${ }^{1,3,4}$ \\ ${ }^{1}$ Indiana University School of Medicine, ${ }^{2}$ Department of Biostatistics, ${ }^{3}$ Indiana University Health, \\ ${ }^{4}$ Regenstrief Institute
}

Background: Adverse maternal and infant health outcomes are influenced by a broad array of psychosocial determinants and behavioral risk factors. We evaluated the correlations between risk factors including mental illness and substance misuse among women from high-risk ZIP Codes in Indianapolis enrolled in a community-based perinatal care program.

Methods: We performed a prospective cohort study of 918 women enrolled in the WeCare community perinatal care program. Data was collected during visits by community health workers (CHWs). CHWs met with the patients at home or over the phone and provided individually tailored care related to mental health, substance use, and nutrition amongst other topics.

Results: Women with anxiety or depression were nearly twice as likely to experience complications during birth (depression: $22.4 \%$ vs. $11.5 \%, p=0.022$; anxiety: $21.9 \%$ vs. $11.2 \%$, $p=0.007$ ). Women with depression were also nearly twice as likely to report substance misuse $(18.4 \%$ vs. $9.6 \%, p=0.046)$ and women with anxiety were more than twice as likely to report substance misuse $(21.9 \%$ vs. $9.0 \%, p=0.0004)$ as well as smoking during pregnancy $(21.9 \%$ vs. $9.7 \%, p=0.0012)$. Women who reported stable or improved anxiety symptoms were less likely to report substance misuse $(8.6 \%$ vs. $19.0 \%, p=0.004)$ and women who reported stable or improved depression symptoms were less likely to report substance misuse ( $8.4 \% \mathrm{vs.} 24.1 \%$, $p=0.0002)$. There was a strong correlation between anxiety and depression. Over $70 \%$ $(71.4 \%)$ of women with depression also presented with anxiety, and over $90 \%(92.2 \%)$ of women who reported stable or improved depression symptoms also reported stable or improved anxiety symptoms.

Conclusions: Many of the risk factors associated with adverse maternal and infant health outcomes exist in interwoven relationships. The inclusion of CHWs into a healthcare team could assist in addressing these factors together in a holistic manner. 